\title{
TROCAS GASOSAS E FLUORESCÊNCIA DA CLOROFILA EM SEIS CULTIVARES DE CAFEEIRO SOB ESTRESSE DE ALUMÍNIO ${ }^{(1)}$
}

\author{
MARIA LUIZA FREITAS KONRAD ${ }^{(2)}$; JOSÉ ALIÇANDRO BEZERRA DA SILVA ${ }^{(3)}$; \\ PEDRO ROBERTO FURLANI ${ }^{(2,4)}$; EDUARDO CARUSO MACHADO ${ }^{(2,4,5)}$
}

\begin{abstract}
RESUMO
Em experimento desenvolvido em casa de vegetação e em câmara de crescimento avaliou-se o efeito do alumínio (Al) na fotossíntese de seis cultivares de cafeeiro. As plantas foram cultivadas em solução nutritiva aerada continuamente, contendo duas concentrações de $\mathrm{Al}, 0$ e 0,148 mmol L-1, fornecidas como $\mathrm{Al}_{2}\left(\mathrm{SO}_{4}\right)_{3}$. Após 97 dias mediram-se as taxas de assimilação de $\mathrm{CO}_{2}(\mathrm{~A})$ e transpiração (E), a condutância estomática (gs), a concentração interna de $\mathrm{CO}_{2}(\mathrm{Ci})$, eficiência instantânea de carboxilação $(\Phi c)$ e variáveis de fluorescência da clorofila. Em todas as cultivares, a presença de Al causou quedas significantes em A, gs, $\Phi$ c, ocorrendo aumento em Ci. Também se observou aumento significativo na fluorescência basal (Fo) e queda na eficiência quântica máxima do fotossistema II (Fv/Fm), sugerindo injúrias na estrutura dos tilacóides causadas pelo Al. Na curva de indução de fotossíntese, observou-se que o Al causou queda no coeficiente de extinção fotoquímica da fluorescência e aumento no coeficiente de extinção não fotoquímico. Os resultados desse estudo indicaram que a queda de A foi devida à queda da condutância estomática, nas atividades bioquímicas e fotoquímicas.
\end{abstract}

Palavras-chaves: Coffea arabica, toxidez de alumínio, fotossíntese, condutância estomática, indução da fotossíntese.

\section{ABSTRACT \\ GAS EXCHANGE AND CHLOROPHYLL FLUORESCENCE IN SIX COFFEE CULTIVARS UNDER ALUMINUM STRESS}

Experiments were carried out under greenhouse and growth chamber to evaluate the effects of aluminum (Al) on several photosynthetic characteristics in six coffee cultivars. Plants were grown in nutrient solution aerated continuously, containing two $\mathrm{Al}$ concentration, 0 and $0.148 \mathrm{mmol} \mathrm{L}^{-1}$, supplied as $\mathrm{Al}_{2}\left(\mathrm{SO}_{4}\right)_{3}$. After 97 days of treatament, measurements of $\mathrm{CO}_{2}$ assimilation rate $(\mathrm{A})$, transpiration rate (E), stomatal conductance (gs), internal $\mathrm{CO}_{2}$ concentration (Ci), instantaneous carboxylation efficiency $(\Phi c)$ and chlorophyll fluorescence related characteristics were performed. All six cultivars showed decrease in A, gs and $\Phi \mathrm{C}$ and increase in Ci. The basal chlorophyll fluorescence yield (Fo) increased and the maximum quantum efficiency $(\mathrm{Fv} / \mathrm{Fm})$ decreased suggesting structural damage in thylakoid. The photosynthetic induction curve revealed that $\mathrm{Al}$ caused decreases in photochemical quenching of fluorescence as well as increases of non-photochemical quenching. Our results indicated that A reduction was related to decreases in both stomatal conductance, and biochemical and photochemical damages.

Key-words: Coffea arabica, aluminum toxicity, photosynthesis, stomatal conductance, photosynthesis induction.

$\left({ }^{1}\right)$ Recebido para publicação em 5 de outubro de 2004 e aceito em 21 de junho de 2005.

$\left({ }^{2}\right)$ Instituto Agronômico (IAC), Caixa Postal 28, 13012-970 Campinas (SP). Brasil. E-mail: 1konrad@brturbo.com; pfurlani@iac.sp.gov.br; caruso@iac.sp.gov.br.

$\left({ }^{3}\right)$ Unviversidade Federal do Vale do São Franciso, Caixa Postal 252, 56304-410 Petrolina (PE). E-mail: jalissandro@bol.com.br

$\left({ }^{4}\right)$ Bolsista de Produtividade em Pesquisa do CNPq.

$\left({ }^{5}\right)$ Autor correspondente. 


\section{INTRODUÇÃO}

A cafeicultura tem-se expandido para as regiões dos cerrados brasileiros onde há grandes áreas de solos com problemas de fertilidade. Entre as limitações do solo, destaca-se a acidez elevada, que favorece a disponibilidade de alumínio (Al) em níveis que podem ser tóxicos para as plantas. A presença e ação tóxica do $\mathrm{Al}$ em solos ácidos prejudicam o crescimento e a produtividade das culturas. O Al acumula-se preferencialmente nas raízes das plantas, sendo parte transportada para a parte aérea. Tal fato talvez justifique que a maior freqüência dos estudos sobre toxidez de Al seja feito em raízes, visto que os efeitos tóxicos se manifestam inicialmente nestes órgãos (Foy et al., 1978). As injúrias que ocorrem nas raízes em função do estresse de $\mathrm{Al}$ são caracterizadas por efeitos sobre a estrutura morfológica, causando engrossamento e diminuindo seu crescimento (Foy et al., 1978). O Al também causa modificações com relação ao metabolismo de minerais, redução da absorção de alguns nutrientes e nos mecanismos de transporte para parte aérea e na sua funcionalidade (Lindon et al., 1999; AKAYA e TAKENAKA, 2001). O Al diminui a atividade respiratória total das raízes (MACHADO e Pereira, 1990). Em cafeeiro sob estresse de Al, essas mesmas características das raízes já foram observadas (Pavan E Binghan, 1982; Braccini et AL., 1998;).

Os efeitos do $\mathrm{Al}$ sobre o crescimento da parte aérea podem ocorrer como conseqüência secundária. A redução do crescimento da planta pode ser resultante da diminuição da atividade fotossintética, que por sua vez pode estar relacionada tanto com fatores estomáticos como não estomáticos. Em algumas espécies, observou-se que a toxidez do Al causa queda na condutância estomática e nas reações bioquímicas de fixação de $\mathrm{CO}_{2}$ (Lindon et al., 1999; Pereira et al., 2000; Akaya e Takenaka, 2001; Рeixoto et al., 2002). Também causa injúrias na formação e na função do cloroplasto (MoustakAs et al., 1996), afetando as membranas do tilacóide e o transporte de elétrons (Moustaka et al., 1995; Pereira et al., 2000; Peixoto et al., 2002) interferindo de forma direta na taxa de assimilação de $\mathrm{CO}_{2}$.

Há evidências que indicam a existência de diferenças quanto à tolerância ao $\mathrm{Al}$, tanto entre espécies como entre cultivares da mesma espécie (FoY et al., 1978). Plantas tolerantes à presença do $\mathrm{Al}$ possuem estratégias que minimizam a toxidez provocada pelo Al (ANDrew e VAn Der BerG, 1973; BRACCini et al., 1998; Peixoto et al., 2002).

Em diversas espécies, verificaram-se respostas diferenciais da taxa de assimilação líquida de $\mathrm{CO}_{2} \mathrm{e}$ de variáveis relacionadas à indução de fluorescência da clorofila em função do grau de tolerância ao Al (Machado e Pereira, 1990; Moustaka et al., 1995; Pereira et al., 2000; Реixoto et al., 2002). A fotossíntese em espécies ou cultivares mais tolerantes é menos afetada; além disso, pode apresentar estratégias de adaptação do aparelho fotossintético em função do tempo de duração do estresse (Регхото et al., 2002). BRACCINI ET AL. (1998) verificaram que em espécies de cafeeiro pode haver diferentes níveis de tolerância em relação à presença do $\mathrm{Al}$ tóxico, quando se avaliam variáveis relacionadas ao crescimento das raízes.

Este trabalho teve como objetivo estudar os efeitos do $\mathrm{Al}$ tóxico sobre as variáveis relacionadas com as trocas gasosas de $\mathrm{CO}_{2}$ e de $\mathrm{H}_{2} \mathrm{O}$ e fluorescência da clorofila em seis cultivares de cafeeiro.

\section{MATERIAL E MÉTODOS}

Utilizaram-se seis cultivares de cafeeiro (Coffea arabica): Bourbom Amarelo (B. Amarelo); Obatã IAC 1669-20 (Obatã); EP395 C.289 - IAC3795 (IAC-3795); Mundo Novo - IAC 388-19 (M. Novo); Icatu Vermelho - IAC 4045 (Icatu) e Catuaí Vermelho 144 (Catuaí).

O experimento foi realizado em casa de vegetação modelo capela com cobertura e laterais de vidro, contendo janelas zenitais laterais e na cumieira. As plantas foram cultivadas em caixas plásticas com capacidade para $15 \mathrm{~L}$ cada uma, contendo solução nutritiva. As sementes dos cafeeiros foram germinadas em tanques de areia lavada e transferidas, no estádio de "orelha-de-onça", para as caixas plásticas contendo solução nutritiva. Cada caixa foi coberta com placas contendo fileiras com furos onde foram colocadas as plântulas de cada cultivar, com suporte de espuma. As plantas foram distribuídas completamente ao acaso. O fornecimento contínuo de oxigênio atmosférico às soluções nutritivas foi feito por meio de um compressor de ar.

A composição da solução nutritiva usada, em mmol L-1 ${ }^{-1}$ com base em Pavan e Binghan (1982), foi a seguinte: $\mathrm{Ca}(1,25) ; \mathrm{Mg}(0,5) ; \mathrm{K}(1,25) ; \mathrm{Fe} \operatorname{EDTA}(0,022)$; $\mathrm{Mn}\left(2,25 \times 10^{-3}\right) ; \mathrm{Cu}\left(1,93 \times 10^{-5}\right) ; \mathrm{Zn}\left(0,48 \times 10^{-4}\right)$; $\mathrm{MoO}_{4}\left(1,30 \times 10^{-4}\right) ; \mathrm{B}(\mathrm{OH})_{4}\left(1,15 \times 10^{-2}\right) ; \mathrm{SO}_{4}(0,5) ; \mathrm{NO}_{3}$ $(3,75) ; \mathrm{P}(0,03)$. Os tratamentos foram o controle sem $\mathrm{Al}$ e o tratamento com $\mathrm{Al}$, cuja concentração foi de $0,148 \mathrm{mmol} \mathrm{L}^{-1}$ na forma de $\mathrm{Al}_{2}\left(\mathrm{SO}_{4}\right)_{3} \cdot 18 \mathrm{H}_{2} \mathrm{O}$. Os valores de $\mathrm{pH}$ das soluções nutritivas, com e sem $\mathrm{Al}$, foram ajustados para $4 \pm 0,2$ com adições de solução $1 \mathrm{mmol} \mathrm{L}^{-1}$ de $\mathrm{H}_{2} \mathrm{SO}_{4}$ ou $\mathrm{KOH}$. A solução nutritiva foi renovada semanalmente e os volumes das caixas, mantidos constantes com adições diárias de água deionizada. 
Aos 97 dias após o transplantio, foram feitas as medidas de trocas gasosas e de fluorescência da clorofila $a$ em seis ou nove plantas por cultivar, por tratamento. Tais plântulas foram transferidas da casa de vegetação para uma câmara de crescimento com controle ambiental.

As medidas da fluorescência da clorofila $a$ foram feitas em folhas localizadas na região intermediária do ramo caulinar por meio de um fluorômetro modulado (PAM-2000, Heinz-Walz). As medidas foram realizadas adotando-se o método do pulso de saturação (SCHreiber et al., 1994) em folhas pré-adaptadas ao escuro em câmara de crescimento $\left(12 \mathrm{~h}\right.$ a $28{ }^{\circ} \mathrm{C}$ e $70 \%$ de umidade relativa). Foram medidos os valores de fluorescência inicial (Fo) e máxima $(\mathrm{Fm})$ após o período de escuro de 12 horas. Com essas variáveis foram estimadas as relações: Fv/ Fo (Pereira et al., 2000) ( $\mathrm{Fv}=\mathrm{Fm}-\mathrm{Fo})$ e a eficiência quântica potencial do fotossistema II (FSII) Fv / Fm, em que Fv é a fluorescência variável $(\mathrm{Fv}=\mathrm{Fm}-\mathrm{Fo})$.

Após as medidas de Fo e Fm, foi efetuada a curva de indução da fotossíntese e análise dos coeficientes de extinção da fluorescência na cultivar Bourbom Amarelo, com quatro repetições. A indução da fotossíntese foi realizada sob densidade de fluxo de fótons fotossinteticamente ativos (DFFFA) de 330 $\mu \mathrm{mol} \mathrm{m} \mathrm{m}^{-2} \mathrm{~s}^{-1}$, durante um período de 30 minutos. Foram calculadas as seguintes variáveis: eficiência quântica efetiva do FSII $\left(\Delta \mathrm{F} / \mathrm{Fm}^{\prime}=\mathrm{Fm}^{\prime}-\mathrm{Fs} / \mathrm{Fm}^{\prime}\right)$, coeficiente de extinção fotoquímico $\left[\mathrm{qP}=\left(\mathrm{Fm}^{\prime}-\mathrm{Fs}\right) /\right.$ $\left.\left(\mathrm{Fm}^{\prime}-\mathrm{Fo}^{\prime}\right)\right]$, coeficiente de extinção não fotoquímico $\left[\mathrm{NPQ}=\left(\mathrm{Fm}-\mathrm{Fm}^{\prime}\right) / \mathrm{Fm}^{\prime}\right]$ e taxa de transporte aparente de elétrons $\left[\mathrm{ETR}=\left(\mathrm{DFFFA}^{*} \Delta \mathrm{F} / \mathrm{Fm}^{\prime}{ }^{*} 0,5 * 0,84\right)\right]$, cujos fatores 0,5 e 0,84 significam fração de excitação distribuída para o FSII e fração de luz absorvida, respectivamente; Fme Fs são fluorescência máxima e no estado de equilíbrio dinâmico, respectivamente, sob luz; Fo' é a fluorescência basal após excitação do fotossistema I (FSI) por radiação infra-vermelha. Os intervalos de medidas variaram entre 15 e 60 s durante um período de 30 minutos (BOLHÀr-NORDENKAMPF e ÖQUIST, 1993). Foram analisados comparativamente os valores de $\Delta \mathrm{F} / \mathrm{Fm}^{\prime}, \mathrm{qP}, \mathrm{NPQ}$ e ETR, durante o período de indução da fotossíntese, nas plantas com e sem estresse de Al.

Após as medidas de fluorescência, as plantas permaneceram na câmara de crescimento por duas horas, nas seguintes condições: $28{ }^{\circ} \mathrm{C}, 350 \mu \mathrm{mol} \mathrm{m}{ }^{-2}$ $\mathrm{s}^{-1}$ de DFFFA, $70 \%$ U.R. Em seguida, foram feitas as medidas de taxa de assimilação líquida de $\mathrm{CO}_{2}(\mathrm{~A})$, condutância estomática (gs), taxa de transpiração (E) e concentração interna de $\mathrm{CO}_{2}(\mathrm{Ci})$ com um sistema portátil de fotossíntese ( $\mathrm{LCi}$, ADC) em folhas submetidas a DFFFA de $900 \mu \mathrm{mol} \cdot \mathrm{m}^{-2} . \mathrm{s}^{-1}$ fornecida por uma lâmpada de halogênio. Foram calculadas as seguintes relações: $\mathrm{WUE}=\mathrm{A} / \mathrm{E}, \mathrm{iWUE}=\mathrm{A} / \mathrm{gs}$ e $\Phi \mathrm{c}=$ A/Ci (ZHANG et al., 2001), em que WUE significa, eficiência do uso de água, iWUE, eficiência intrínseca do uso de água e $\Phi c$, eficiência instantânea de carboxilação.

O delineamento experimental adotado foi em blocos casualizados. Foram utilizadas seis repetições para as medidas de trocas gasosas, nove para as medidas de fluorescência e quatro para as medidas de indução da fotossíntese. Os dados foram submetidos à análise de variância (teste F) e comparação de médias pelo teste de Tukey a $5 \%$ de probabilidade.

\section{RESULTADOS E DISCUSSÃO}

\section{Trocas gasosas}

Quando se considerou o cultivo das plantas em solução nutritiva sem $\mathrm{Al}$ os valores de $\mathrm{A}$ das seis cultivares de cafeeiros não diferiram estatisticamente, apesar de apresentarem pequenas diferenças, enquanto para gs e E somente Obatã obteve maiores valores $(p<0,05)$ quando não submetidos ao estresse por Al (Figura 1). Os valores observados de A, gs e E estão dentro da faixa de variação constante na literatura para cafeeiros (FAHL et al., 2001; SiLVA et al., 2004; Alfonsi et al., 2005). Em todas as cultivares submetidas ao estresse de Al ocorreu queda significante em $A(p<0,05)$ quando comparadas com o controle (Figura 1A). Os valores relativos de A nos cafeeiros com e sem estresse de $\mathrm{Al}$ (razão entre $\mathrm{A}_{\text {com }}$ $\mathrm{Al} / \mathrm{A}_{\text {sem Al }}$ ) foram: 0,35 em IAC3795; 0,30 em M. Novo, 0,31 em Icatu; 0,34 em Catuai; 0,57 em B. Amarelo e 0,43 em Obatã. Portanto, as cultivares B. Amarelo e Obatã foram relativamente menos afetadas que as demais $(p<0,05)$. Apesar dessa diferença na resposta de $A$ não se deve considerar essas duas cultivares (Catuai e B. Amarelo) como tolerantes, visto que a queda em A também foi acentuada, respectivamente, $43 \%$ e $57 \%$. Em quatro tipos de porta-enxerto de citros cultivados em presença de alumínio, na concentração de $0,4 \mathrm{~mol} \mathrm{~L}^{-1}$, foram verificadas quedas em A entre $55 \%$ e $85 \%$ em relação ao controle (PEREIRA et al., 2000). Comparando-se a fotossíntese de cultivares consideradas tolerantes e sensíveis de sorgo na presença de 0,185 $\mathrm{mmol} \mathrm{L}^{-1}$ de $\mathrm{Al}$ (Регхото et al, 2002), de milho na presença de $4,5 \mathrm{~g} \mathrm{~L}^{-1}$ e de arroz na presença de $9,0 \mathrm{~g} \mathrm{~L}^{-1}$ de $\mathrm{Al}$ na solução nutritiva (Machado e Pereira, 1990), após 14 dias sob estresse, foram observadas quedas diferenciais entre cultivares consideradas tolerantes e sensíveis. Em plantas de diversas espécies e diferentes cultivares de uma mesma espécie pode haver diferenças quanto a resposta de $\mathrm{A}$ à presença tóxica do $\mathrm{Al}$. 


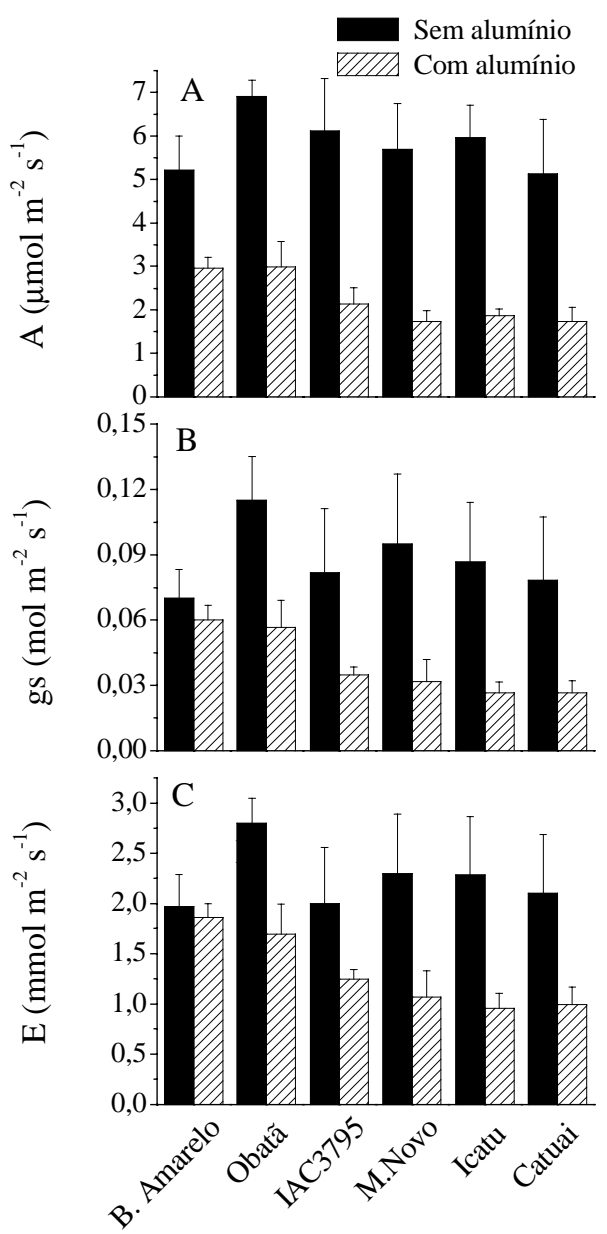

Figura 1. (A) Taxa de assimilação de $\mathrm{CO}_{2}$ (A); (B) condutância estomática (gs) e (C) taxa de transpiração (E) em seis cultivares de cafeeiro [Bourbom Amarelo (B. Amarelo), Obatã IAC 1669-20 (Obatã) , EP395 C.289 - IAC3795 (IAC-3795), Mundo Novo - IAC 388-19 (M. Novo), Icatu Vemelho - IAC 4045 (Icatu) e Catuaí Vermelho 144 (Catuaí)] cultivados com e sem presença de $0,148 \mathrm{mmol} . \mathrm{L}^{-1}$ de $\mathrm{Al}$ em solução nutritiva. Barras indicam desvio-padrão da média.

Exceto em B. Amarelo, gs e E diminuíram significativamente em função do estresse de Al. A queda nos valores de gs e E, em função do estresse de $\mathrm{Al}$, foi similar à de $\mathrm{A}$ nas respectivas cultivares (Figura 1). Em termos relativos E decresceu menos que A com a queda de gs, ou seja, as razões $E_{\text {com Al }} / E_{\text {sem }}$ Al foram: 0,62 em IAC3795; 0,47 em M. Novo; 0,42 em Iacatu; 0,47 em Catuaí; 0,95 em B. Amarelo e 0,6 para Obatã. Dessa forma, WUE também diminuiu com a presença do Al na solução nutritiva, sendo significativamente menor que nas plantas sem $\mathrm{Al}$ (Figura 2A). Em citros, PEREIRA et al. (2000) verificaram queda em WUE em plantas sob estresse de $\mathrm{Al}$; no entanto, essa queda ocorreu em virtude do aumento de E em paralelo ao decréscimo de A. Em citros, nas espécies analisadas, não houve queda em gs (PEREIRA et al., 2000), ao contrário do observado para cafeeiro em que gs sempre diminuiu com a presença de Al. O mesmo também foi verificado em trigo (MoustaKas et al., 1996), sorgo (Реіхото et al., 2002) e Quercur glauca (AKAYA e TAKENAKa, 2001).

Na relação entre A/gs, que define a eficiência intrínseca do uso de água (iWUE), também se observou queda significante nos cafeeiros submetidos ao estresse de $\mathrm{Al}(\mathrm{p}<0,05)$ (Figura 2B). iWUE define que, para uma abertura estomática equivalente, o cafeeiro submetido ao estresse de $\mathrm{Al}$ possui menor valor de A; as diminuições dessas duas relações, WUE e iWUE, sugerem que a queda em gs foi apenas um dos fatores que contribuíram para queda de $\mathrm{A}$ nas cultivares estudadas, excetuando-se B. Amarelo em que não houve queda em gs. Outros autores também observaram que a queda de gs contribuiu para a queda de A em plantas submetidas a esse tipo de estresse (Реiхото et al., 2002; AKAYA e TAKENAKA, 2001), todavia outros fatores como o metabolismo fotossintético (limitação bioquímica) e transporte eletrônico (limitação fotoquímica) podem contribuir para queda de A em plantas sob estresse de Al (Lindon et al., 1999; PEREIRA et al., 2000; AKaya e TAKenaKa, 2001; Peixoto et al, 2002).
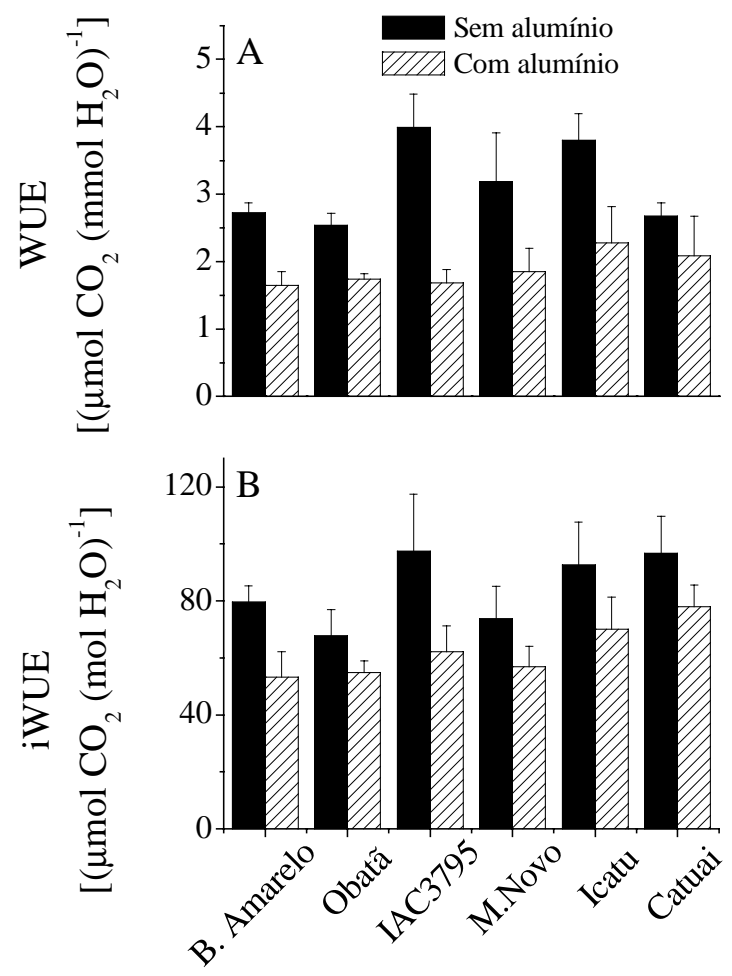

Figura 2. (A) Eficiência do uso de água (WUE) e eficiência intrínsica do uso de água (iWUE) em seis cultivares de cafeeiro [Bourbom Amarelo (B. Amarelo), Obatã IAC 166920 (Obatã) , EP395 C.289 - IAC3795 (IAC-3795), Mundo Novo - IAC 388-19 (M. Novo), Icatu Vemelho - IAC 4045 (Icatu) e Catuaí Vermelho 144 (Catuaí)], cultivados com e sem presença de $0,148 \mathrm{mmolL}^{-1}$ de $\mathrm{Al}$ em solução nutritiva. Barras indicam desvio-padrão da média. 
Caso não ocorra queda na atividade metabólica da fixação de $\mathrm{CO}_{2,}$ é esperada uma redução na concentração interna de $\mathrm{CO}_{2}(\mathrm{Ci})$ quando gs diminui (RAsCHKE, 1979). Verificou-se, entretanto, que $\mathrm{Ci}$ aumentou significativamente $(\mathrm{p}<0,05)$ em todas as cultivares de cafeeiro em função da presença de $\mathrm{Al}$ na solução nutritiva (Figura $3 \mathrm{~A}$ ) a despeito da diminuição de gs (Figura 1B). O aumento médio em

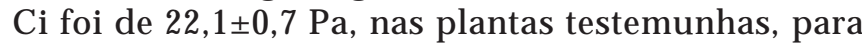
$25,9 \pm 0,4 \mathrm{~Pa}$ nas plantas submetidas ao estresse de $\mathrm{Al}$ (Figura 3A). Em B. Amarelo e Catuaí a diferença entre $\mathrm{Ci}$ com e sem $\mathrm{Al}$ foi mais pronunciada. Esse resultado corrobora com a sugestão de que o decréscimo em A nas plantas sob estresse de Al também foi ocasionado pela queda da atividade fotossintética da folha. Também em Quercus glauca (AKAYA e TAKENAKA, 2001), em sorgo (Peixoto et al., 2002), em citros (Pereira et al., 2000) e trigo (MoustAKAS et al., 1996) demonstrouse que a queda em A foi decorrente tanto de fatores relacionados com o estômato como com o metabolismo fotossintético. De fato, no caso presente, os valores de Фc (Figura 3B) dos cafeeiros sob estresse foram significativamente menores que nos cafeeiros sem estresse, revelando que a eficiência instantânea de carboxilação foi afetada pela presença de Al.

\section{Fluorescência da clorofila $a$}

Quando as plantas são expostas a estresse ambiental ou biótico, alterações no estado funcional das membranas dos tilacóides dos cloroplastos provocam mudanças nas características dos sinais de fluorescência, os quais podem ser quantificados nas folhas (Ribeiro et al, 2003; BAKer e Rosenqvst, 2004). A fluorescência tem sido utilizada para identificar cultivares tolerantes ao Al (MoustaKAs et al., 1995). As variáveis $\mathrm{Fo}, \mathrm{Fm}, \mathrm{Fv} / \mathrm{Fm}$ e $\mathrm{Fv} / \mathrm{Fo}$ relacionadas à fluorescência da clorofila $a$ dos cafeeiros não submetidos ao estresse de $\mathrm{Al}$ foram iguais entre si ( $\mathrm{p}$ $<0,05$ ) (Figura 4) à semelhança do que ocorreu com as variáveis relacionadas às trocas gasosas (Figuras $1,2$ e 3$)$.

Nos cafeeiros submetidos ao estresse de $\mathrm{Al}$, tais variáveis foram significativamente afetadas. Fo nas plantas com Al foi, em média 1,93 vezes maior quando comparadas às plantas sem Al. Somente em Obatã, Fo das plantas sob estresse foi maior que nas demais cultivares. Fo é a fluorescência quando $Q_{A}$ (quinona receptora primária de elétrons do FSII) está totalmente oxidada e o centro de reação do FSII está aberto, situação iminente à ativação das reações fotoquímicas. O aumento de Fo, que portanto é independente dos eventos fotoquímicos, reflete destruição do centro de reação do FSII ou diminuição na capacidade de transferência da energia de excitação da antena para o centro de reação (BAKER e Rosengvst, 2004). Assim, em todas as cultivares avaliadas o FSII foi afetado pelo Al. O aumento de Fo foi acompanhado por aumento médio de $22 \%$ de Fm nos cafeeiros sob estresse de $\mathrm{Al}$, em relação ao controle. Fm indica a intensidade máxima de fluorescência que ocorre quando praticamente toda $\mathrm{Q}_{\mathrm{A}}$ está reduzida e os centros de reação são incapazes de aumentar as reações fotoquímicas, atingindo sua capacidade máxima (BAKER e RosenQvST, 2004). A razão Fv/Fm dos cafeeiros com Al decresceu, em média, 13\% em relação aos controles. No entanto, não houve diferença entre as cultivares, sendo as testemunhas iguais entre si e as plantas com Al também iguais entre si.
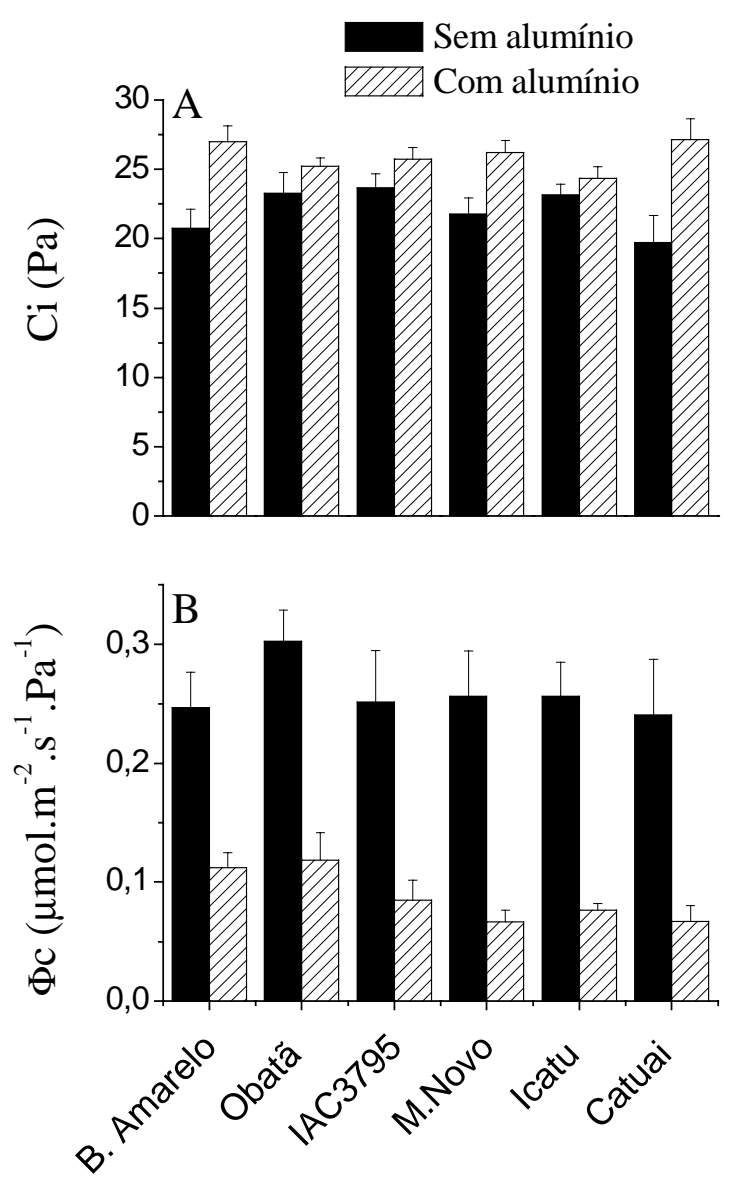

Figura 3. (A) Concentração interna de $\mathrm{CO}_{2}(\mathrm{Ci})$; (B) eficiência intantânea de carboxilação $\left(\Phi_{C}\right)$ em seis cultivares de cafeeiro [Bourbom Amarelo (B. Amarelo), Obatã IAC 1669-20 (Obatã), EP395 C.289 - IAC3795 (IAC-3795), Mundo Novo - IAC 388-19 (M. Novo), Icatu Vemelho - IAC 4045 (Icatu) e Catuaí Vermelho 144 (Catuaí)], cultivados com e sem presença de 0,148 mmol $\mathrm{L}^{-1}$ de $\mathrm{Al}$ em solução nutritiva. Barras indicam desviopadrão da média. 

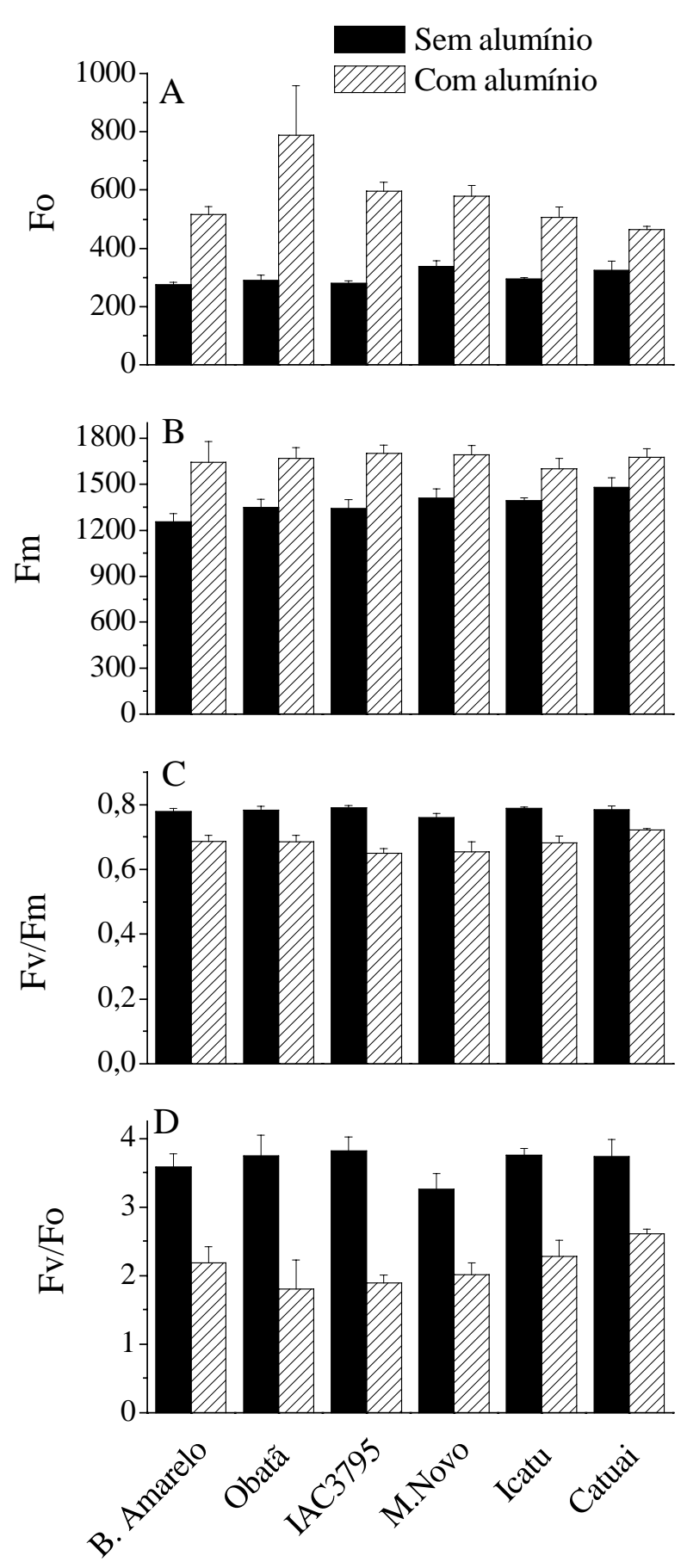

Figura 4. (A) Fluorescência basal (Fo) e (B) máxima (Fm) da clorofila $a$; (C) eficiência quântica potencial do FS II $(\mathrm{Fv} / \mathrm{Fm})$ e (D) razão Fv/Fo em seis cultivares de cafeeiro [Bourbom Amarelo (B. Amarelo), Obatã IAC 1669-20 (Obatã) , EP395 C.289 - IAC3795 (IAC-3795), Mundo Novo - IAC 388-19 (M. Novo), Icatu Vemelho - IAC 4045 (Icatu) e Catuaí Vermelho 144 (Catuaí)], cultivados com e sem presença de $0,148 \mathrm{mmol}^{-1} \mathrm{~L}^{-1} \mathrm{de}$ $\mathrm{Al}$ em solução nutritiva. Barras indicam desvio-padrão da média.
Em sorgo (Регхото et al., 2002), em citros (Pereira et al., 2001) e trigo (Moustakas et al., 1997), comparando cultivares tolerantes com sensíveis observaram reduções significantes de $\mathrm{Fv} / \mathrm{Fm}$ nas plantas sensíveis. Nos cafeeiros, a queda em Fv/Fm foi semelhante em todas as cultivares, não ocorrendo discriminação. Fv/Fm é uma estimativa da eficiência quântica máxima da atividade fotoquímica do FSII, quando todos os centros de reação do FSII estão abertos (BAKER e RosenQvst, 2004). Essa relação tem sido utilizada freqüentemente para detectar perturbações no sistema fotossintético causada por estresses ambientais e bióticos, visto que a diminuição indica inibição da atividade fotoquímica. Decréscimos em Fo e Fv/Fm também foram observados em trigo, sorgo e citros (Moustakas et al., 1997; Регхото et al., 2002; Pereira et al., 2000). Pereira et al. (2000) e Peixoto et al. (2002) utilizaram a razão Fv/Fo para detectar estresse de $\mathrm{Al}$ em citros e em sorgo, respectivamente, pois essa razão amplifica as pequenas variações detectadas em Fv/Fm, como pode ser observado na comparação entre as duas razões na Figura 4C e 4D. Nesse caso, a razão Fv/Fo em cafeeiros com Al decresceu $42 \%$ em relação aos controles. No entanto, mesmo utilizando essa relação, não se verificou diferenças significantes nas seis cultivares avaliadas neste estudo, ou seja, nessas cultivares foram observadas a mesma resposta em relação ao estresse de $\mathrm{Al}$, não se discriminando cultivares com diferentes tolerâncias ao Al tóxico. Em espécies, porém, com diferentes níveis de tolerância verificaram-se diferentes razões Fv / Fo (BAKER e ROSENQVST, 2004).

Na Figura 5, observa-se a indução da fotossíntese quando a planta foi transferida do escuro para a luz; nesse processo, os centros de reação do FSII são progressivamente reduzidos, nos primeiros segundos, ocorrendo um progressivo aumento da fluorescência da clorofila. Em seguida, a fluorescência decai em um fenômeno chamado extinção da fluorescência (quenching) que pode ser fotoquímica e não fotoquímica. A extinção fotoquímica (qP) é iniciada em função do aumento dos elétrons exportados do FSII devido à indução da ativação de enzimas envolvidas no metabolismo do carbono e da abertura estomática. Ao mesmo tempo, ocorre aumento na extinção não fotoquímica (NPQ), ou seja, aumento na proporção de energia utilizada na geração de $\mathrm{DpH}$ transtilacoidal (BAKer e Rosenqvist, 2004). Sob condições constantes de luz e temperatura, a variação destes dois processos foi completada no período entre 15 e 30 minutos. A indução da fotossíntese e os coeficientes de extinção da fluorescência são apresentados na Figura 5, para a cultivar B. Amarelo, submetida ou não ao estresse de Al. 

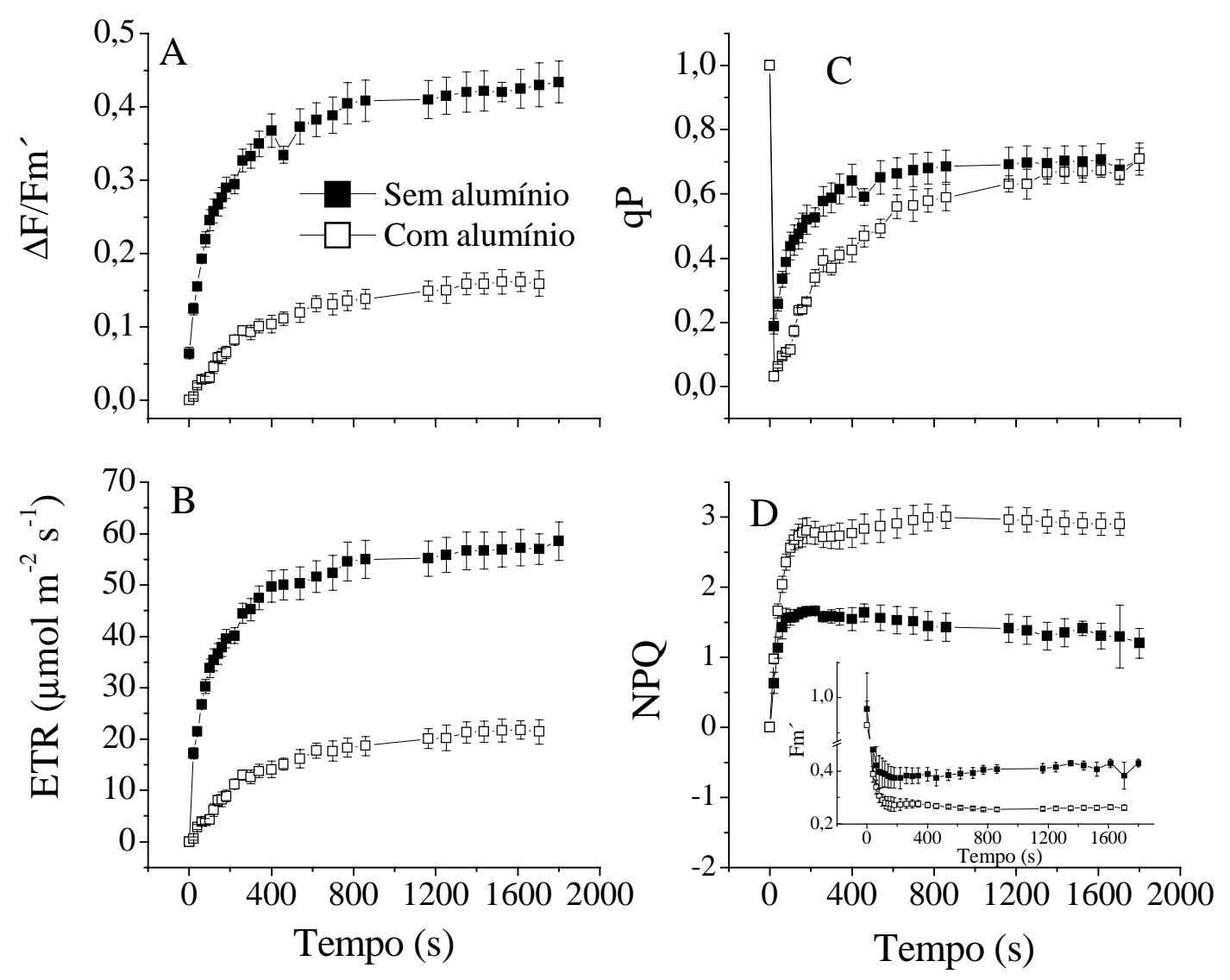

Figura 5. Curva de indução da fotossíntese em cafeeiro cultivar Bourbom Amarelo cultivado com e sem presença de 0,148 $\mathrm{mmol} \mathrm{L}^{-1}$ de Al em solução nutritiva. (A) Eficiência quântica efetiva do FS II ( $\Delta$ F/Fm'); (B) taxa aparente de transporte de elétrons (ETR); (C) coeficiente de extinção fotoquímica da fluorescência (qP); (D) coeficiente de extinção não fotoquímica da fluorescência (NPQ); inserido em (D) fluorescência máxima à luz. Barras indicam desvio-padrão da média.

A eficiência quântica efetiva, descrita pela razão $\Delta \mathrm{F} / \mathrm{Fm}^{\prime}$, indica qual a proporção de luz que foi absorvida pela clorofila associada ao FSII utilizada em atividade fotoquímica, como tal, informa a quantidade de elétrons transportados sendo um indicativo da fotossíntese (BAKER e ROSENQVST, 2004). O principal fator determinante desta eficiência é a habilidade com que os elétrons são removidos da quinona receptora do FSII, que é diretamente relacionado com a taxa de consumo dos produtos do transporte fotossintético de elétrons (ATP e NADPH). No cafeeiro sob estresse de Al ocorreu queda significativa dessa eficiência, e também de A nas plantas sob estresse (Figura 1A). O decréscimo na eficiência de carboxilação em cafeeiros sob estresse de $\mathrm{Al}$ (Figura 3B) pode ser um dos fatores que afetam a taxa de consumo de ATP e NADPH (BAKER e RosenQvST, 2004). Tal restrição no consumo de elétrons pode resultar em aumento do NPQ e decréscimo em $\Delta \mathrm{F} / \mathrm{Fm}^{\prime}$ (Figura 5A e D). Fm' foi particularmente sensível ao tratamento com Al. Nas plantas controle, alto valor de Fm' associado com baixo NPQ indicam maior eficiência de utilização da energia no Ciclo de Calvin. Sob condições de laboratório, como foi desenvolvido este experimento, há uma relação linear entre eficiência de utilização de energia e fixação de $\mathrm{CO}_{2}$, sendo alguns desvios nesta relação ocasionados em condições que proporcionam mudança na taxa de fotorrespiração (MAXWELl e JoHNSON, 2000).

A figura $5 \mathrm{~B}$ descreve o produto entre $\Delta \mathrm{F} / \mathrm{Fm}^{\prime}$ e DFFFA, ou seja, o transporte aparente de elétrons (ETR). Tal índice possui a mesma dinâmica da curva de resposta de A em função de DFFFA (BILGER et al., 1995). Durante a indução da fotossíntese, observouse em $\Delta \mathrm{F} / \mathrm{Fm}^{\prime}$ e ETR um padrão semelhante, de forma consistente com a maior taxa de assimilação de $\mathrm{CO}_{2}$ das plantas sem estresse de $\mathrm{Al}$ em relação às com estresse (Figura 1 e 5B), ou seja, o Al causou queda substancial no transporte de elétrons para fixação de $\mathrm{CO}_{2}$ em B. Amarelo. 
Nos coeficientes de extinção da fluorescência, qP e NPQ houve respostas diferentes em relação às plantas com e sem Al (Figuras 5C e D); qP foi maior no cafeeiro sem $\mathrm{Al}$, principalmente no início do período de indução até ao redor dos 6 minutos iniciais, sugerindo que a ativação das enzimas de carboxilação foi mais rápida. Depois houve tendência dessa diferença diminuir (Figura 5C). Maior qP revelou que a extinção da fluorescência para processos fotoquímicos foi maior nas plantas sem estresse, ou que mais energia foi extinta em processos primários das reações fotoquímicas (KRAUSE E WeIS, 1991). Valores maiores ou constantes de qP em paralelo ao decréscimo de A indicam aumento da participação de dreno alternativo de elétrons, como a fotorrespiração (Schreiber e Bilger, 1987; Ribeiro et al., 2004). Assim, no momento em que qP das plantas com estresse de $\mathrm{Al}$ tende a atingir o mesmo patamar que das plantas sem estresse é possível que esteja ocorrendo um aumento na participação da fotorrespiração na extinção fotossintética da fluorescência.

\section{CONCLUSÕES}

1. Nas cultivares de cafeeiro utilizadas neste trabalho não houve diferenças importantes quanto à tolerância ao $\mathrm{Al}$, visto que em todas elas o processo da fotossíntese foi afetado significativamente e em intensidades semelhantes.

2. A presença de $\mathrm{Al}$ causou queda em gs e em A. Apesar de gs ter diminuído Ci aumentou devido à queda na eficiência de carboxilação. $\mathrm{O} \mathrm{Al}$ causou queda em Fv/Fm e aumento em Fo, indicando destruição da estrutura das membranas do tilacóides. A queda na taxa de assimilação de $\mathrm{CO}_{2}$ em função do Al foi acompanhada de queda no coeficiente de extinção fotoquímico e aumento no coeficiente não fotoquímico da fluorescência. Assim em cafeeiro, a queda da taxa de assimilação de $\mathrm{CO}_{2}$, devida ao efeito tóxico do Al, está relacionada tanto a fatores estomáticos (queda de gs) como bioquímicos e por injúrias na atividade fotoquímica do aparato fotossintético.

\section{REFERÊNCIAS}

ALFONSI, E. L.; FAHL, J.L.; CARELLI, M. L. C.; FAZUOLI, L.C. Crescimento, fotossíntese e composição mineral em genótipos de Coffea com potencial para utilização como porta-enxerto. Bragantia, Campinas, v. 64, p.1- 13, 2005.

AKAYA, M.; TAKENAKA, C. Effects of aluminum stress on photosynthesis of Quercus glauca Thrumb. Plant and Soil, London, v.237, p.137-146, 2001.
ANDREW, C.S.; VAN DER BERG, P.J. The influence of aluminum on phosphate sorption by whole plants and excised roots of some pasture legumes. Plant and Soil, London, v.24, p.351-349, 1973.

BAKER, N.R.; ROSENQVST, E. Applications of chlorophyll fluorescence can improve crop production strategies: an examination of future possibilities. Journal of Experimental Botany, Oxford, v.55, p.1607-1621, 2004.

BILGER, W.; SCHREIBER U.; BOCK M. Determination of the quantum efficiency of photosystem II and non-photochemical quenching of chlorophyll fluorescence in the field. Oecologia, v.102, p.425-432, 1995.

BOLHÀR-NORDENKAMPF HR, ÖQUIST GO. Chlorophyll fluorescence as a tool in photosynthesis research. In: Hall, D.O.; SCURLOCK, J.M.O.; BOLHÀR-NORDENKAMPF. H.R.; LEEGOOD, R.C., LONG, S.P. (Ed.). Photosynthesis and production in a changing environment: a field and laboratory manual. London: Chapman e Hall, 1993. p.193-206.

BRACCINI, M.C.L., MARTINEZ, H.E.P., PEREIRA, P.R.G. SAMPAIO, N.F. e SILVA, E.A.M. Tolerância de genótipos de cafeeiro ao Al em solução nutritiva. I. Crescimento e desenvolvimento da parte aérea e sistema radicular. Revista Brasileira de Ciências do Solo, Campinas, v.22, p.435-442, 1998.

FAHL, J.I.; CARELLI, M.L.; MENEZES, H.C.; GALLO, P.B.; TRIVELLIN, P.C.O. Gas exchange, growth, yield and beverage quality of Coffea arabica cultivars grafted to C. canephora and C. congensis. Experimental Agriculture, Cambridge, v.37, p.241$252,2001$.

FOY, C. D.; CHANEY, R.L.; WHITE, M.C. The physiology of metal toxicity in plants. Annual Review of Plant Physiology, Boca Raton, v.29, p.511-566, 1978.

KRAUSE, G.H.; WEIS, E.. Chlorophyll fluorescence and photosynthesis: the basics. Annual Review of Plant Physiology and Plant Molecular Biology, Boca Raton, v.42, p.313-349, 1991.

LINDON, F.C.; RAMALHO, J.C.; BARREIRO, M.G.; LAURIANO, J.A. Modulation of photosystem 2 reactions mediated by aluminium toxicity in Zea mays. Photosynthetica, Prague, v.34, p.151-156, 1999.

MACHADO, E.C.; PEREIRA, A.R. Eficiência de conversão e coeficiente de manutenção da planta inteira, das raízes e da parte aérea em milho e arroz submetidos ao estresse de Al. Pesquisa Agropecuária Brasileira, Brasília, v.25, p.845-855, 1990.

MAXWELL, K.; JOHNSON, G.N. Chlorophyll fluorescence: a pratical guide. Journal of Experimental Botany, Oxford, v.51, p.659-668, 2000.

MOUSTAKA, M; OUZOUNIDOU, G.; LANNOYER, R. Aluminum effects on photosynthesis and elemental uptake in an aluminum-tolerant and non-tolerant wheat cultivar. Journal of Plant Nutrition, New York, v.18, p.669-683, 1995. 
MOUSTAKA, M; OUZOUNIDOU, G.; LANNOYER, R. Indirect effects of aluminum stress on function of the photosynthetic apparatus. Plant Physiology and Biochmistry, Paris, v.34, p.553-569, 1996.

MOUSTAKAS, M.; ELEFTHERIOU, E.P.; OUZOUNIDOU, G. Short-term effects of aluminum at alkaline $\mathrm{pH}$ on the structure and function of the photosynthetic apparatus. Photosynthetica, Prague, v.34, p.169-177, 1997.

PAVAN, M.A.; BINGHAM, F. T. Toxidez de Al em cafeeiros cultivados em solução nutritiva. Pesquisa Agropecuária Brasileira, Brasília, v.17, p.1293-1302, 1982.

PEIXOTO, P.H.P. ; DA MATTA, F.M. ; CAMBRAIA, J. Responses of the photosynthetic apparatus to aluminum stress in two sorghum cultivars. Journal of Plant Nutrition, New York, v.25, p.821-832, 2002.

PEREIRA, W.E.; SIQUEIRA, D.L.; MARTINEZ, C.A.; PUIATTI, $M$. Gas exchange and chlorophyll fluorescence in four citrus rootstocks under aluminium stress. Journal of Plant Physiology, v.157, p.513-520, 2000.

RASCHKE, K. Movements using turgor mechanisms: Movements of stomata. In: HAUPT, W.; FEINLEIB, M.E. (Ed.). Encyclopedia of plant physiology. Berlin: Springer-Verlag, 1979. v.7, p.383-441.

RIBEIRO, R.V.; MACHADO, E.C.; OLIVEIRA, R.F. Early photosynthetic responses of sweet orange plants infected with Xylella fastidiosa. Physiological and Molecular Plant Pathology, London, v.62, p.167-173, 2003.
RIBEIRO, R.V.; MACHADO, E.C.; OLIVEIRA, R.F. Growth and leaf temperature effects on photosynthesis of sweet orange plants infected with Xylella fastidiosa. Plant Pathology, Oxford, v.53, p. 334-340, 2004.

SCHREIBER, U.; BILGER, W. 1987. Rapid assessment of stress effects on plant leaves by chlorophyll fluorescence measurements. In: TENHUNEN JD, CATARINO FM, LANGE OL, OECHEL WC. (Ed.). Plant response to stress. Berlin: Springer-Verlag, 1987. p.2753. (NATO ASI Series, Series G)

SCHREIBER, U.; BILGER, W.; NEUBAUER, C. Chlorophyll fluorescence as a nonintrusive indicator for rapid assessment of in vivo photosynthesis. In: SCHULZE, E.D.; CALDWELL, M.M. (Ed.). Ecophysiology of photosynthesis. Berlin: Springer, 1994. p.49-70. (Ecological Studies, 100)

SILVA, E.A.; DA MATTA, F.M.; DUCATTI, A.J.; BARROS, R.S. Seasonal changes in vegetative growth and photosynthesis of Arábica coffee trees. Field Crops Research, Amsterdam, v. 89, p. 349-357, 2004.

ZHANG, S.; LI, Q.; MA, K.; CHEN, L. Temperaturedependent gas exchange and stomatal/non-stomatal limitation to $\mathrm{CO}_{2}$ assimilation of Quercus liaotungensis under midday higher irradiance. Photosynthetica, Prague, v.39, p. 383-388, 2001. 